\title{
Comprehensive Analysis and Experimental Validation of an Improved Mathematical Modeling of Photovoltaic Array
}

\author{
Satarupa Bal, ${ }^{1}$ Anup Anurag, ${ }^{2}$ Mrutyunjaya Nanda, ${ }^{3}$ and Suman Sourav ${ }^{4}$ \\ ${ }^{1}$ Department of Electrical and Computer Engineering, National University of Singapore, 4 Engineering Drive 3, Singapore 117583 \\ ${ }^{2}$ Department of Electrical Engineering and Information Technology, ETH Zurich, Rämistrasse 101, 8092 Zurich, Switzerland \\ ${ }^{3}$ Department of Electrical Engineering, Asian Institute of Technology, Pathumthani 12120, Thailand \\ ${ }^{4}$ School of Computing, National University of Singapore, 13 Computing Drive, Singapore 117417
}

Correspondence should be addressed to Anup Anurag; anup.rana123@gmail.com

Received 26 August 2014; Revised 30 November 2014; Accepted 1 December 2014

Academic Editor: Jose Antenor Pomilio

Copyright (C) 2015 Satarupa Bal et al. This is an open access article distributed under the Creative Commons Attribution License, which permits unrestricted use, distribution, and reproduction in any medium, provided the original work is properly cited.

\begin{abstract}
This paper proposes a simple, accurate, and easy to model approach for the simulation of photovoltaic (PV) array and also provides a comparative analysis of the same with two other widely used models. It is highly imperative that the maximum power point (MPP) is achieved effectively and thus a simple and robust mathematical model is necessary that poses less mathematical complexity as well as low data storage requirement, in which the maximum power point tracking (MPPT) algorithm can be realized in an effective way. Further, the resemblance of the $P-V$ and $I-V$ curves as obtained on the basis of experimental data should also be taken into account for theoretical validation. In addition, the study incorporates the root mean square deviation (RMSD) from the experimental data, the fill factor (FF), the efficiency of the model, and the time required for simulation. Two models have been used to investigate the $I-V$ and $P-V$ characteristics. Perturb and Observe method has been adopted for MPPT. The MPP tracking is realized using field programmable gate array (FPGA) to prove the effectiveness of the proposed approach. All the systems are modeled and simulated in MATLAB/Simulink environment.
\end{abstract}

\section{Introduction}

Electrical energy from photovoltaic is currently regarded as the prerequisite sustainable resource for both stand-alone as well as grid connected applications, since it is abundant and clean, offers zero input fuel cost, and is distributed throughout the earth [1]. In practical cases, photovoltaic modules operate over a highly intermittent nature of temperature and irradiance but the electrical parameters provided in the datasheet are only for the standard test conditions (STC). Moreover, in power generation from PV, optimal utilization of the available solar energy is imperative due to the high costs of PV modules. It is also seen that mathematical models of few individual components of PV system are represented and simulated for better understanding of their performances [2].

This calls for a simple, accurate, and easy to model approach for the simulation of photovoltaic (PV) module to track the maximum power point and to predict $\mathrm{PV}$ energy production under varying atmospheric conditions [3]. In order to increase the accuracy, the following can be incorporated, but it leads to the increase in complexity of the modeling [4]:

(i) temperature dependence of the diode saturation current,

(ii) temperature dependence of the photo current,

(iii) inclusion of series resistance: for more accurate shape between the MPP and the open circuit (OC) voltage,

(iv) inclusion of shunt resistance in parallel with the diode,

(v) variability of diode quality factor,

(vi) introduction of two or more parallel diodes.

The accuracy of the simulation of a PV model largely depends on the estimation of the characteristic $I-V$ and $P-V$ 
curves. Furthermore, factors such as efficiency, field factor, and simulation time affect the effectiveness of the model. A simplistic and easy to model approach is preferred so as to avoid unwanted complexity due to additional parameters.

So far, among the mathematical models of PV array proposed in the literature, the simplest is the ideal single diode model which involves only three parameters, namely, short circuit current, open circuit voltage, and the diode ideality factor [5]. Improvement has been made, with the simplified single diode model (SSDM) being proposed in [6] which takes the effect of the series resistance $\left(R_{s}\right)$ which is the sum of several types of structural resistance of the device into consideration [7-15]. The influence of $R_{s}$ only becomes dominant when the PV device operates in the voltage source region. Also, it lacks the accuracy when subjected to large temperature variations [16]. Since, the value of $R_{s}$ is very low, some authors neglect its effect [5, 17-19]. Further improvement has been done by the introduction of the single diode model (SDM) which includes the additional shunt resistance $\left(R_{p}\right)$ along with the series resistance [2]. The shunt resistance exists mainly due to the leakage current of the p-n junction. The effect of $R_{p}$ is dominant when the PV device operates at current source region of operation. However, since the value of $R_{p}$ is very high many authors $[4,8,9,20,21]$ neglect it in order to simplify the model. Although it is much more accurate than the previous models, it is not preferred on account of its computational complexity. It is also reported in [16] that the accuracy of this model deteriorates at low irradiance levels. In order to mitigate the inaccuracies offered by the previous models, the two-diode model was proposed in [22]. However, this leads to more model complexity and thus more simulation time due to the involvement of a greater number of parameters. A new mathematical PV model has also been proposed in [23] that includes the advantages of previous models combining the three main considerations, namely, simplicity, ease of modeling, and accuracy. However, it doesnt take into consideration the effect of diode saturation current on temperature which results in model errors at the vicinity of open-circuit voltage and consequently at other regions.

This paper proposes a new, simple, accurate, and easy to model approach for the simulation of PV array and also provides a comparative analysis of the same with the conventional single diode model and the improved ideal single diode model. As the PV systems are generally integrated with specific control algorithms in order to extract the maximum possible power, it is highly imperative that the MPP is achieved effectively and thus it is needed to design a model from which the MPPT algorithm can be realized in an effective way. Some MPPT techniques have been proposed in $[1,3,4,10]$. However, for simplicity, this paper adopts the Perturb and Observe (P\&O) method for MPPT.

The proposed theoretical model is verified and validated with experimental data of commercial PV array. RMSD from the experimental data, maximum efficiency of the design, the fill factor (FF), and the simulation time has also been calculated. In addition, the MPP tracking is realized in digital environment using FPGA kit to prove the effectiveness of the proposed approach. All the systems here are modeled and simulated in MATLAB/Simulink environment. The proposed modeling method can be useful for users who require simple, fast, and accurate models in simulation of PV systems.

\section{Mathematical Models for a Photovoltaic Module}

The major issue of real-time identification is basically the selection of a proper model. It is therefore necessary to have a proper mathematical model that can represent accurately the current-voltage characteristics of the PV array and which can be solved by analytical methods in a simplified manner [24]. In addition to this to maximize the power extracted from a PV array with the help of MPPT control, the understanding and modeling of PV cell are also important [25].

Assuming the semiconductor diode equation and the Kirchhoff laws, the $I-V$ characteristics for a PV module composed of series connected cells based on single exponential model are expressed as follows [26]:

$$
I_{l}=I_{p v}-I_{0}\left[\exp \left(\frac{q\left(V+I_{l} R_{s}\right)}{N_{s} A k T}-1\right)\right]-\left(\frac{V+I_{L} R_{s} N_{s}}{N_{S} R_{p}}\right),
$$

where $k$ is the Boltzmann constant $\left(1.3806 \times 10^{-23} \mathrm{~J} / \mathrm{K}\right)$ and $q$ the electron charge $\left(1.60217 \times 10^{-19} \mathrm{C}\right) . T$ gives the module temperature. The parameter $I_{p v}$ gives the photocurrent, $I_{0}$ represents the diode saturation current, and $I_{l}$ gives the output current. $R_{s}$ and $R_{p}$ give the series resistance and the shunt resistance. $A$ and $N_{s}$ represent the diode ideality factor and the number of cells connected in series, respectively.

The first term $I_{p v}$ gives the photocurrent and the second part is the ideal dark current that models the emitter and base recombination. All the parameters are mostly calculated through sets of nonlinear equations [27].

2.1. Single Diode Model (SSDM). The single diode model takes into account both the series resistance as well as the shunt resistance unlike the ideal single diode model or the simplified single diode model as shown in Figure 1. This resistance is the sum of several types of structural resistance of the device. $R_{s}$ depends mainly on four factors, namely [2],

(i) contact resistance of the metal base with p-layer,

(ii) resistance of p-layer and n-layer,

(iii) contact resistance of the metal grid with the n-layer,

(iv) resistance of the grid.

The shunt resistance exists mainly due to the leakage current of the $\mathrm{p}-\mathrm{n}$ junction. It depends basically on the fabrication method of the PV cell.

Mathematically, the current equation can be written in accordance with Kirchhoff's current law:

$$
I_{p v}=I_{d}+I_{p}+I_{l}
$$

where $I_{p v}$ is the photocurrent generated due to the incident light and $I_{d}$ is the diode current (Shockley diode equation). 


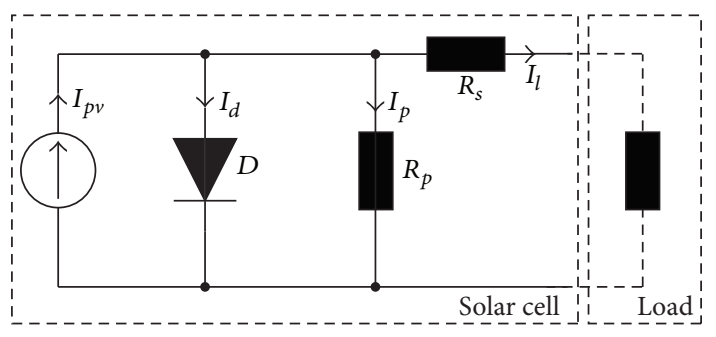

Figure 1: PV cell modeled as single diode model circuit.

$I_{l}$ gives the output current and there is an additional term, $I_{p}$ which represents the leakage current of the p-n junction. In this model, the diode is given by

$$
I_{d}=I_{0}\left[\exp \left(\frac{q\left(V+I_{l} R_{s}\right)}{N_{s} A k T}-1\right)\right]
$$

where the module saturation current $\left(I_{0}\right)$ is given by

$$
I_{0}(T)=\frac{I_{\text {scr }}\left(T_{\text {ref }}\right)+K_{i} \Delta T}{\exp \left[q\left(V_{\text {oc }}\left(T_{\text {ref }}\right)+K_{v} \Delta T\right) / A k T\right]},
$$

where $I_{\text {scr }}$ is the PV module short circuit current (in A) and can be found in the product datasheet. $K_{i}$ is the short circuit current temperature coefficient (in $\mathrm{A} / \mathrm{K}$ ). Thus, from (3) and (4) the diode current can be calculated. Now, $I_{p v}$ can be calculated by

$$
I_{p v}=G \times\left[I_{\mathrm{scr}}+K_{i} \Delta T\right],
$$

where $G$ is the PV module incident illumination $\left(\right.$ in $\mathrm{kW} / \mathrm{m}^{2}$ ). An additional current $I_{p}$ is introduced here:

$$
I_{p}=\frac{V+N_{s} I_{l} R_{s}}{N_{s} R_{p}} .
$$

Therefore, there are three basic unknown parameters: $A, R_{s}$, and $R_{p}$. Here, the value of $A$ is assumed $(1.1<A<1.6)$. The value of the resistance can be calculated from the information available in the datasheet.

For the calculation of the series resistance and the shunt resistance, an iterative method is employed. Some authors vary the resistance independent of each other leading to poor accuracy of results. Here, a concept of simultaneously varying both the values is shown. The idea is taken from the fact that the maximum calculated power should be equal to the power mentioned in the datasheet. From (2), (3), and (6), the current equation can be written and the equation at MPP gives the value of $R_{p}$ to be

$$
R_{p}=\frac{V_{\mathrm{mpp}}\left(V_{\mathrm{mpp}}+N_{s} I_{\mathrm{mpp}} R_{s}\right)}{N_{s}\left(V_{\mathrm{mpp}}\left(I_{p v}-I_{d, \mathrm{mpp}}\right)-P_{\max }\right)},
$$

where

$$
I_{d, \mathrm{mpp}}=I_{0}\left[\exp \left(\frac{q\left(V_{\mathrm{mpp}}+I_{\mathrm{mpp}} R_{s}\right)}{N_{s} A k T}\right)\right] .
$$

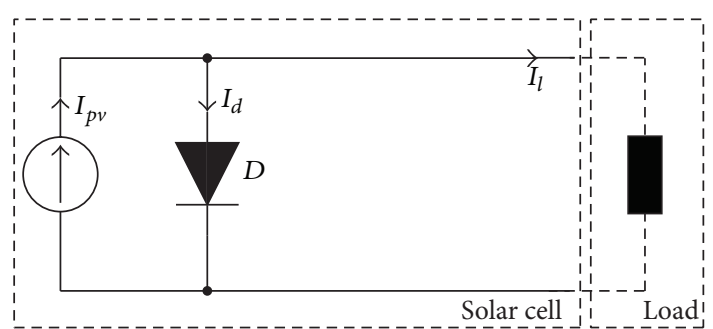

FIGURE 2: PV cell modeled as improved ideal single diode model circuit.

The value of $I_{0}$ can be calculated as from (4) and $I_{p v}$ can be calculated from (5). However, some simplifications have to be made in order to find the unknown parameters. An initial guess is taken as

$$
\begin{gathered}
R_{s, \text { initial }}=0, \\
R_{p \text {,initial }}=\frac{V_{\mathrm{mpp}}}{I_{\mathrm{scr}}\left(T_{\mathrm{ref}}\right)-I_{\mathrm{mpp}}}-\frac{V_{\mathrm{oc}}\left(T_{\mathrm{ref}}\right)-V_{\mathrm{mpp}}}{I_{\mathrm{mpp}}} .
\end{gathered}
$$

Using an iterative procedure, by increasing the value of the series resistance and simultaneously updating the shunt resistance so as to match the maximum power, the output current equation can be found out. The major drawback of this model lies on its assumption of ideality factor. Also, the computational complexity and number of iterations required to obtain the output current equation are considerable.

2.2. Improved Ideal Single Diode Model. The improved ideal single diode model is basically based on the ideal single diode model as shown in Figure 2. However, the modeling involves a set of mathematical equations which produces sufficiently accurate results but with much reduced complexity.

The series and shunt resistances are neglected for mathematical simplicity. However, the method of deriving the parameters is of reduced complexity. The computation of these equations avoids the use of a nonlinear solver [28].

The current relation can be found by applying KCL:

$$
I_{p v}=I_{d}+I_{l} \text {. }
$$

Here, the current through the diode is given by

$$
I_{d}=I_{0}\left[\exp \left(\frac{q V}{A k T}\right)-1\right] \text {. }
$$

The derivation of the saturation current $I_{0}$ begins by

$$
V_{\text {oc }}(G, T)-V_{\text {oc }}\left(G, T_{\text {ref }}\right)=-|\beta| \Delta T,
$$

where $V_{\mathrm{oc}}(G, T)$ and $V_{\mathrm{oc}}\left(G, T_{\mathrm{ref}}\right)$ represent the open circuit voltages at a temperature $T$ and at the reference temperature $T_{\text {ref }} \beta$ gives the voltage temperature coefficient and that can be found from the product datasheet. The open circuit voltage can be found out by putting $I_{l}=0$ in (10) and equating the value of $I_{d}$ as in (11):

$$
V_{\mathrm{oc}}=\frac{A k T}{q} \ln \left(\frac{I_{p v}}{I_{0}}+1\right) .
$$




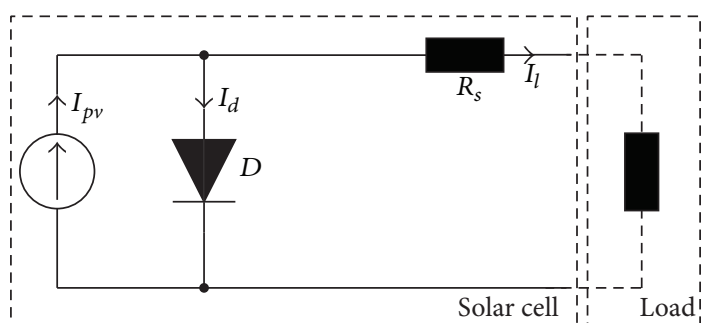

Figure 3: PV cell modeled as improved simplified single diode model circuit.

Now, finding the values of $V_{\mathrm{oc}}$ at both the temperatures using (13) and replacing in (12) the following is obtained:

$$
\begin{aligned}
\frac{k A}{q}[ & T \ln \left(\frac{G\left(I_{\mathrm{scr}}+K_{i} \Delta T\right)}{I_{0}}+1\right) \\
& \left.-T_{\text {ref }} \ln \left(\frac{G I_{\mathrm{scr}}}{I_{0}\left(T_{\text {ref }}\right)}+1\right)\right]=-|\beta| \Delta T .
\end{aligned}
$$

Rearranging (14), the following is found:

$$
I_{0}=\frac{\exp (q|\beta| \Delta T / A k T) \times G \times\left(I_{\mathrm{scr}}+K_{i} \Delta T\right)}{\left(G I_{\mathrm{scr}} / I_{0}\left(T_{\mathrm{ref}}+1\right)^{T_{\mathrm{ref}} / T}\right)-\exp (q|\beta| \Delta T / A k T)} .
$$

The $I_{0}\left(T_{\text {ref }}\right)$ can be calculated according to (4). Now, writing (11) at MPP,

$$
I_{\mathrm{mpp}}=I_{p v}-I_{0}\left[\exp \left(\frac{q V_{\mathrm{mpp}}}{A k T}\right)-1\right],
$$

and here according to (5), at reference temperature,

$$
I_{p v}=I_{\mathrm{scr}}
$$

The ideality factor A can be derived by substituting (15) and (17) into (16):

$$
\frac{I_{\mathrm{mpp}}}{I_{\mathrm{scr}}}=\exp \left(\frac{q V_{\mathrm{mpp}}}{A k T_{\mathrm{ref}}}\right)-\left(\frac{I_{\mathrm{scr}}-I_{\mathrm{mpp}}}{I_{\mathrm{scr}}}\right) \exp \left(\frac{q V_{\mathrm{oc}}}{A k T_{\mathrm{ref}}}\right) \text {. }
$$

Thus, the unknown parameters can be found out from the above equations and from the product datasheet information.

However, the temperature dependence of the saturation current has not been considered. Also, there is a slight deviation of the $I-V$ characteristics from the experimental curve, due to the approximation $R_{s}=0$.

2.3. Improved $P V$ Modeling Approach. In the proposed improved PV modeling approach, in order to avoid the complexity offered by the single diode model, it relies on the simplified single diode model, by neglecting the shunt resistance, as shown in Figure 3. Applying KCL to the above circuit, the current relation is found to be the same as (10).

The diode current equation incorporates the additional voltage drop across the series resistance:

$$
I_{d}=I_{0}\left[\exp \left(\frac{q\left(V+I_{l} R_{s}\right)}{A k T}\right)-1\right] .
$$

$I_{p v}$ is calculated according to (5). The current equation for the simplified single diode model is given by

$$
I_{l}=I_{p v}-I_{0}\left[\exp \left(\frac{q\left(V+I_{l} R_{s}\right)}{A k T}\right)-1\right] .
$$

Now, there are basically four unknown parameters: $I_{p v}, I_{0}, A$, and $R_{s} . I_{p v}$ can be determined from the information available in the manufacturer s datasheet by applying (5).

The value of the series resistance can be calculated from the information available in the product datasheet. Since, the current at maximum power point at reference temperature is available in the product datasheet, (20) is solved for maximum power point conditions:

$$
I_{\mathrm{mpp}}=I_{p v}-I_{0}\left[\exp \left(\frac{q\left(V_{\mathrm{mpp}}+I_{\mathrm{mpp}} R_{s}\right)}{A k T}\right)-1\right] .
$$

Thus, the series resistance can be found from

$$
R_{s}=\frac{(A k T / q) \times \ln \left(\left(I_{p v}-I_{\mathrm{mpp}}\right) / I_{0}+1\right)-V_{\mathrm{mpp}}}{I_{\mathrm{mpp}}} .
$$

In order to find the absolute value of all these parameters, the ideality factor and the saturation current should be calculated effectively. In the earlier works, the value of the ideality factor has been assumed which leads to the degradation of the curve. This model has the novelty of calculating the value of the ideality factor from the datasheet parameters so that the simulated curves coincide with the experimental data to a larger extent. Also, an expression has been provided for finding the saturation current from the information given in the datasheet.

For the calculation of the ideality factor, advantage has been taken of the fact that the derivative of power with respect to voltage at MPP is zero.

Differentiating (20) with respect to voltage gives

$$
\begin{aligned}
\frac{d I_{l}}{d V}=-I_{0}[ & \exp \left(\frac{q v}{A k T}\right) \times \frac{q R_{s}}{A k T} \times \exp \left(\frac{q I_{l} R_{s}}{A k T}\right) \times \frac{d I_{l}}{d V} \\
& \left.+\exp \left(\frac{q I_{l} R_{s}}{A k T}\right) \times \frac{q}{A k T} \times \exp \left(\frac{q V}{A k T}\right)\right] .
\end{aligned}
$$

Now, it is known at MPP the derivative power with respect to voltage is zero. And hence the derivative of load current with respect to voltage is given by

$$
\left.\frac{d I_{l}}{d V}\right|_{\mathrm{MPP}}=-\frac{I_{\mathrm{mpp}}}{V_{\mathrm{mpp}}} .
$$

So, at MPP,

$$
\begin{aligned}
-\frac{I_{\mathrm{mpp}}}{V_{\mathrm{mpp}}}=-I_{0}[ & \exp \left(\frac{q V}{A k T}\right) \times \frac{q R_{s}}{A k T} \times \exp \left(\frac{q I_{l} R_{s}}{A k T}\right) \times-\frac{I_{\mathrm{mpp}}}{V_{\mathrm{mpp}}} \\
& \left.+\exp \left(\frac{q I_{l} R_{s}}{A k T}\right) \times \frac{q}{A k T} \times \exp \left(\frac{q V}{A k T}\right)\right] .
\end{aligned}
$$




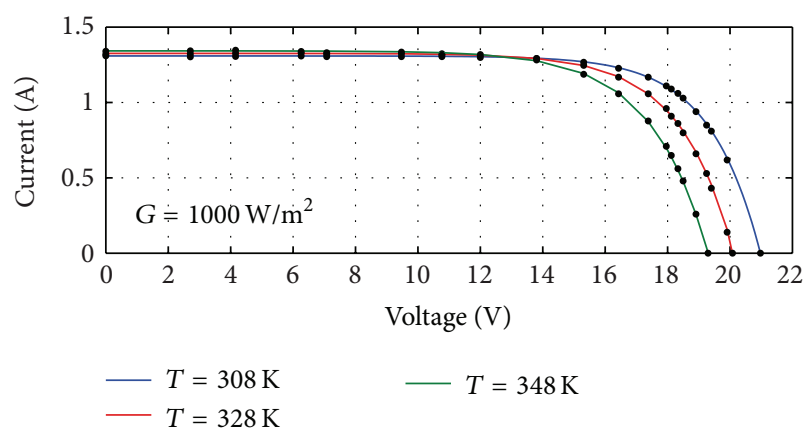

(a)

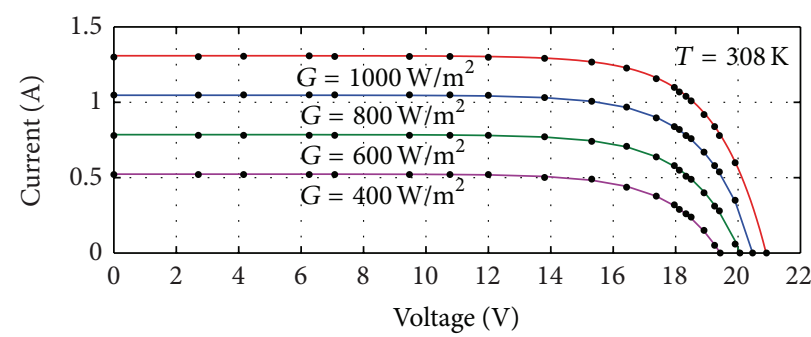

(b)

FIGURE 4: (a) $I-V$ model curves with experimental data for TBP-1237 solar array at different temperatures and (b) $I$ - $V$ model curves with experimental data for TBP-1237 solar array at different irradiations.

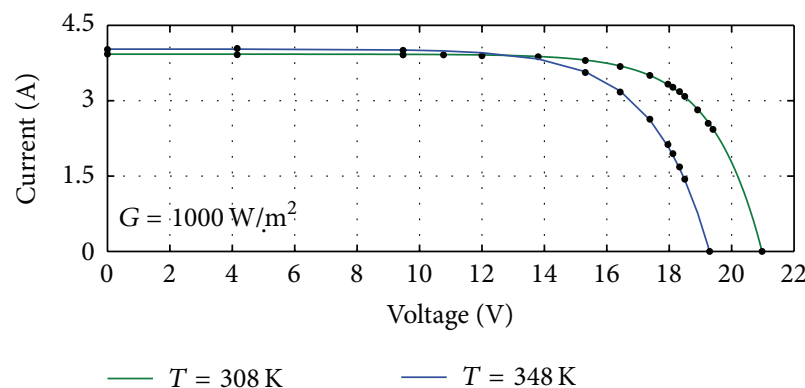

(a)

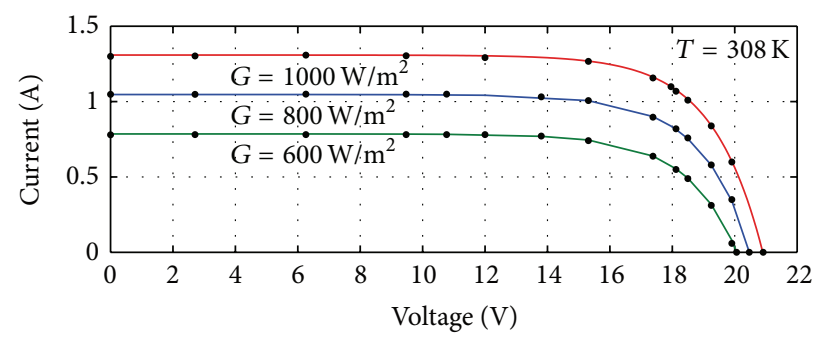

(b)

Figure 5: (a) $I-V$ model curves with experimental data for MSX60 solar array at different temperatures and (b) $I$ - $V$ model curves with experimental data for MSX60 solar array at different irradiations.

Thus, the value of the saturation current equals

$$
\begin{aligned}
& I_{0}(T) \\
& =I_{\mathrm{mpp}} \\
& \quad \times\left(V_{\mathrm{mpp}} \times\left[\exp \left(\frac{q V}{A k T}\right) \times \frac{q R_{s}}{A k T} \times \exp \left(\frac{q I_{l} R_{s}}{A k T}\right) \times-\frac{I_{\mathrm{mpp}}}{V_{\mathrm{mpp}}}\right.\right. \\
& \left.\left.\quad+\exp \left(\frac{q I_{l} R_{s}}{A k T}\right) \times \frac{q}{A k T} \times \exp \left(\frac{q V}{A k T}\right)\right]\right)^{-1} .
\end{aligned}
$$

The reverse saturation current is given by (4). Equating (4) and (26) at reference temperature

$$
\begin{gathered}
\frac{I_{\mathrm{scr}}\left(T_{\mathrm{ref}}+K_{i} \Delta T\right)}{\exp \left[q\left(V_{\mathrm{oc}}\left(T_{\mathrm{ref}}\right)+K_{v} \Delta T\right) / A k T\right]} \\
=I_{\mathrm{mpp}} \times\left(V_{\mathrm{mpp}} \times\left[\exp \left(\frac{q V}{A k T}\right) \times \frac{q R_{s}}{A k T} \times \exp \left(\frac{q I_{l} R_{s}}{A k T}\right)\right.\right. \\
\times-\frac{I_{\mathrm{mpp}}}{V_{\mathrm{mpp}}}+\exp \left(\frac{q I_{l} R_{s}}{A k T}\right) \\
\left.\left.\times \frac{q}{A k T} \times \exp \left(\frac{q V}{A k T}\right)\right]\right)^{-1},
\end{gathered}
$$

$R_{s} / A$ can be obtained from (22) and is replaced in (27). Now, the equation has only one unknown parameter $A$. Also, the saturation current can be calculated from (26).

This modification aims at smoothing the curve between the MPP and the open circuit point and also to match the open circuit voltages for a large range of temperature variations.

\section{Validating the Model}

The PV array model is simulated in the MATLAB/ Simulink environment in order to validate the claims. Tables 1 and 2 give a comparative view on the parameters on the datasheet and the parameters obtained from the proposed simulation model. It is seen that the three main points coincide with that of the datasheet. The characteristic curves developed from the model have been plotted with the experimental data at four different temperature conditions and three different irradiation values. This has been done so as to effectively verify the notion that the proposed model gives acceptable results even if the conditions are considerably far from STC as shown in Figures 4 and 5.

Figure 4 gives the $I-V$ curves for TBP-1237 at different temperatures and different irradiations, respectively.

However, in order to test the validity efficiently, it is required to test the model with other solar panels. Figures 
TABLE 1: Parameters of TBP-1237 solar module at STC (Temp: $25^{\circ} \mathrm{C}$; A.M.: $1.5 ; G=1000 \mathrm{~W} / \mathrm{M}^{2}$ ).

\begin{tabular}{lccc}
\hline Parameter & Label & $\begin{array}{c}\text { Value from } \\
\text { datasheet }\end{array}$ & $\begin{array}{c}\text { Value from } \\
\text { model }\end{array}$ \\
\hline Maximum power & $P_{\max }$ & $20 \mathrm{~W}$ & $20 \mathrm{~W}$ \\
Open circuit voltage & $V_{\mathrm{oc}}$ & $20.91 \mathrm{~V}$ & $20.91 \mathrm{~V}$ \\
Short circuit current & $I_{\mathrm{scr}}$ & $1.3 \mathrm{~A}$ & $1.3 \mathrm{~A}$ \\
Voltage at MPP & $V_{\mathrm{mpp}}$ & $17.1 \mathrm{~V}$ & $17.1 \mathrm{~V}$ \\
Current at MPP & $I_{\mathrm{mpp}}$ & $1.17 \mathrm{~A}$ & $1.17 \mathrm{~A}$ \\
\hline
\end{tabular}

TABle 2: Parameters of MSX60 solar module at STC (Temp: $25^{\circ} \mathrm{C}$; A.M.: $1.5 ; G=1000 \mathrm{~W} / \mathrm{M}^{2}$ ).

\begin{tabular}{lccc}
\hline Parameter & Label & $\begin{array}{c}\text { Value from } \\
\text { datasheet }\end{array}$ & $\begin{array}{c}\text { Value from } \\
\text { model }\end{array}$ \\
\hline Maximum power & $P_{\max }$ & $59.85 \mathrm{~W}$ & $59.85 \mathrm{~W}$ \\
Open circuit voltage & $V_{\mathrm{oc}}$ & $21.1 \mathrm{~V}$ & $21.1 \mathrm{~V}$ \\
Short circuit current & $I_{\mathrm{scr}}$ & $3.8 \mathrm{~A}$ & $3.8 \mathrm{~A}$ \\
Voltage at MPP & $V_{\mathrm{mpp}}$ & $17.1 \mathrm{~V}$ & $17.1 \mathrm{~V}$ \\
Current at MPP & $I_{\mathrm{mpp}}$ & $3.5 \mathrm{~A}$ & $3.5 \mathrm{~A}$ \\
\hline
\end{tabular}

5(a) and 5(b) give the $I-V$ curves for MSX60 at different temperatures and different irradiations, respectively.

3.1. Effect of Variation in Temperature and Irradiation. It can be seen that the values of the parameters found can be used for irradiation and temperature close to the STC. With a large change in the temperature or irradiation, a small modification of the algorithm can be put forward to account for the change in the model parameters.

Although there is an increase of the photo current with the increase in temperature increase owing to the slight decrease of the band gap energy, $E_{g}$, the main output characteristics, such as efficiency, are negatively influenced by high temperature. The series resistance increases with increase in temperature. However, a decrease is observed with the increase in irradiation [28]. On the other hand, the junction characteristic parameters such as ideality factor and saturation current are highly temperature dependent. The variation of ideality factor is almost linear with temperature. It increases with increase in temperature [29]. This is explained from the fact that, at the increased temperatures, imperfections of basic material are more pronounced and thus create defects in the lattice structure.

The short-circuit current from a solar cell depends linearly on light intensity which results in the increase in PV output power as the solar radiation increases. The series resistance is basically derived from the slope of the $I-V$ curve between the MPP and the open-circuit point. It is hence treated as fitting parameter rather than fixed panel s effective series resistance.

The slope is strongly dependent on the panel short-circuit current and maximum power point current and therefore also on the irradiation. Hence, it tends to increase with increase in light intensity.
It has to be pointed out that the variation of the parameters with respect to temperature can be accounted for the calculation of $I_{p v}$ directly from (5). The dependence of resistance with respect to temperature has been shown in (22). However, the change in the series resistance and the ideality factor of the modeling method can be accounted for. The series resistance can also be given by [30]

$$
R_{s}=2\left(\frac{V_{\mathrm{oc}}}{I_{\mathrm{sc}}}-\frac{\mathrm{Ar}}{I_{\mathrm{sc}}^{2}}-\frac{A V_{t}}{I_{\mathrm{sc}}}\right)
$$

The values of $V_{\mathrm{oc}}$ and $I_{\mathrm{sc}}$ can be determined at any given temperature and irradiation according to [31]. Ar gives the area under the curve. Now, an iterative procedure is done so as to find the exact values for the ideality factor and the series resistance. This can be described as Figure 6. " $e$ " can be defined as any small value close to zero. The ideality factor shows a linear behavior and, thus, it can be calculated manually by the linear nature as in [29] for the practical working temperatures:

$$
\frac{A}{A_{\mathrm{STC}}}=\frac{T}{T_{\mathrm{STC}}},
$$

where $A_{\mathrm{STC}}$ and $T_{\mathrm{STC}}$ are the ideality factor and the temperature at STC. This model thus proves to be an efficient model as compared to the previous approaches, even though it neglects the shunt resistance, on account of the fact that all the parameters are found from the datasheet parameters, instead of taking any assumptions into account. The improved ideal single diode model also finds the parameters but it neglects the series resistance which leads to increased deviation from the experimental values. Since, none of the parameters are assumed; this approach provides a better model than its predecessors, which rely on intelligent assumptions so as to fit the curve.

\section{Comparative Results and Analysis}

With the purpose of comparison between the three different approaches of mathematical modeling of PV array and validating them experimentally, a small-scale module of has been considered. The accuracy of the model is experimentally validated using TBP-1237 and MSX60. The proposed PV circuit model is implemented using a current controlled source and simple computational blocks. The inclusion of series resistance and ideality factor as unknown parameters aids in the smoothness of the output characteristics between MPP and open-circuit voltage and, thus, the curve coincides closely with the experimental data obtained not only at the three main points but throughout the region. Table 3 gives the components used in the prototype. For experimental purpose, three arrays of bulbs with three bulbs of $200 \mathrm{~W}$ in each row are used as artificial sun. The conventional DCDC boost converter with a control tracker is implemented for MPP tracking.

4.1. Analysis on Basis of $P-V$ and $I-V$ Curves. The $I-V$ and $P-V$ output curves for different mathematical models are 


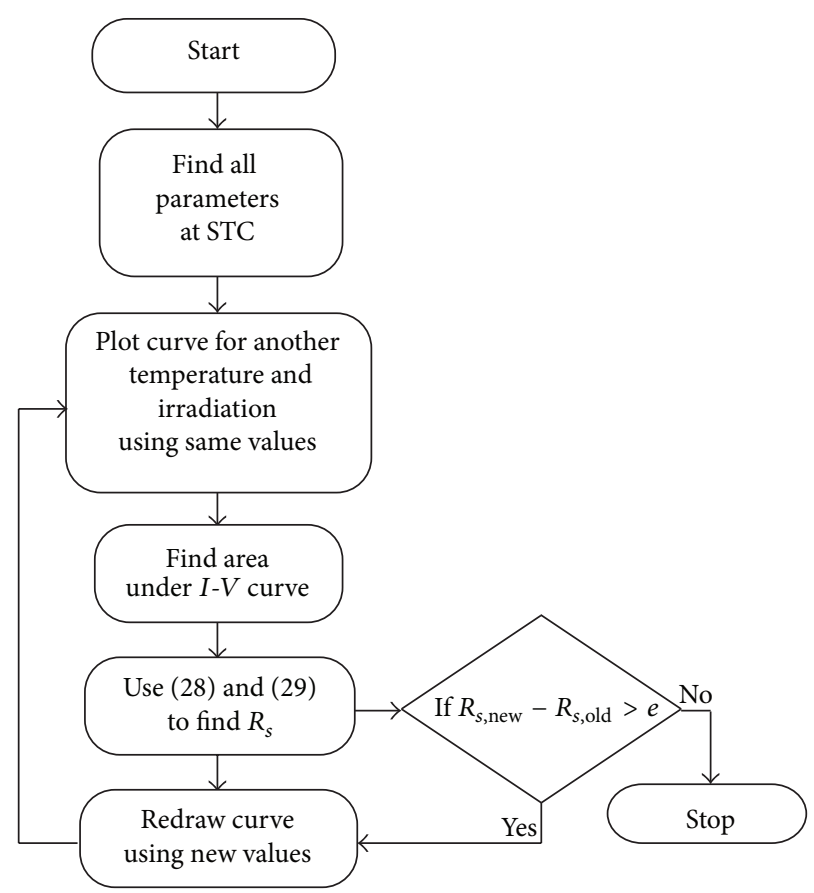

FIGURE 6: Flowchart for determining the fianl values of the parameters at any temperature and irradiation.

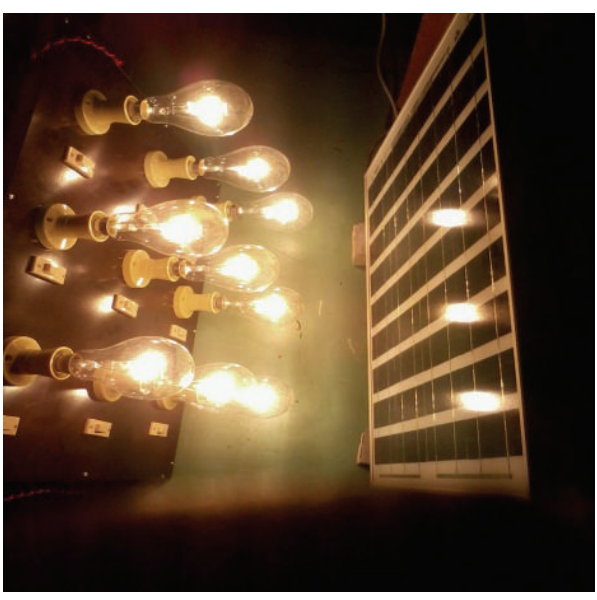

(a)

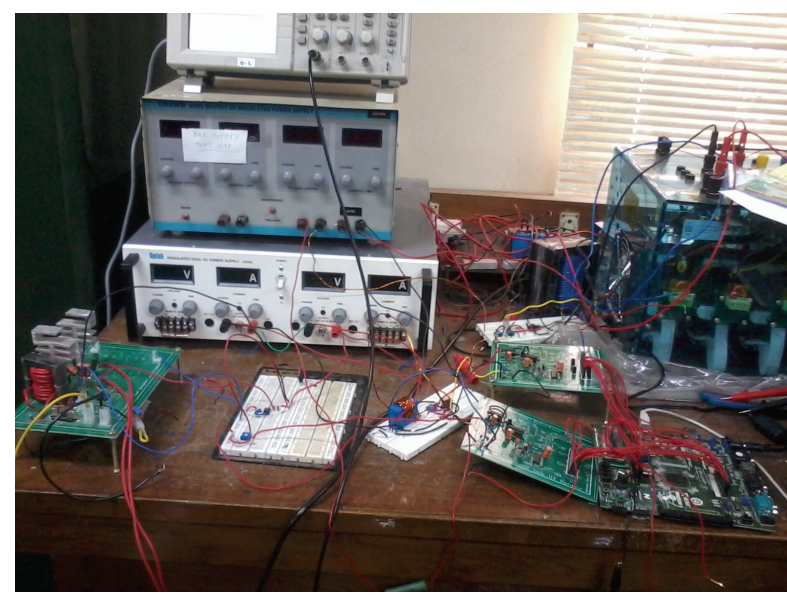

(b)

Figure 7: (a) Bulbs used as artificial sun for PV panel and (b) prototype of the conventional boost converter used.

TABLE 3: Components used in the study.

\begin{tabular}{lcc}
\hline Parameter & Label & Value/equipment \\
\hline Inductance & $L$ & $1.7 \mathrm{mH}$ \\
Output capacitor & $C_{\text {out }}$ & $300 \mu \mathrm{F}$ \\
Load & $G D$ & IR2213 \\
A to D converter & ADC & THS1030 \\
Current sensor & CS & LEM LA-55P \\
\hline
\end{tabular}

compared with the experimental results for determining the accuracy of the models and their closeness to the actual curve. For the single diode model, the graph obtained coincides with the experimental result approximately at $I_{\mathrm{sc}}$ and $V_{\mathrm{oc}}$ but the MPP deviates from the one obtained from experimental result. For the improved ideal single diode model, the simulation result at MPP points exhibit less deviation with improvement in $V_{\text {oc }}$ point as compared to the former. It takes advantage of the simplicity of ideal models and enhances the accuracy by deriving a mathematical representation, capable of extracting accurate estimates of the model parameters, directly related to manufacturer datasheets. The inclusion of the calculation of ideality factor affects the curvature of the $I-V$ curve and thus expresses the ideality of the diode. The mathematical derivation of the same improves the model fitting. However, owing to the assumption of no series or shunt resistance, the model shows some deviation 


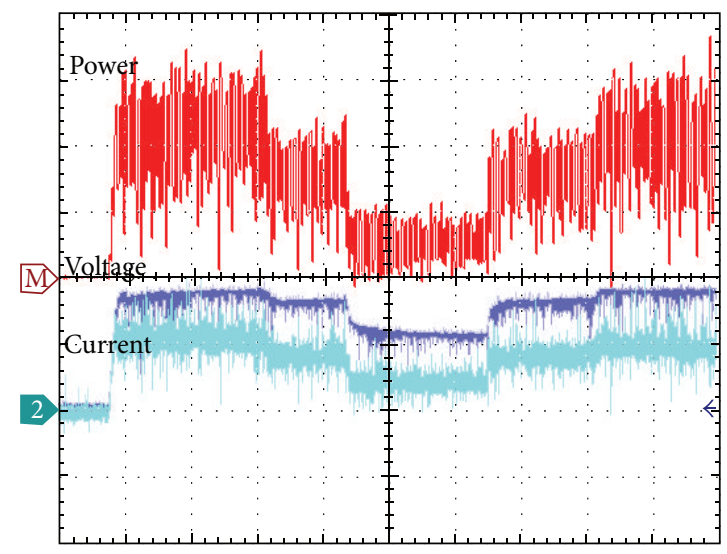

(a)

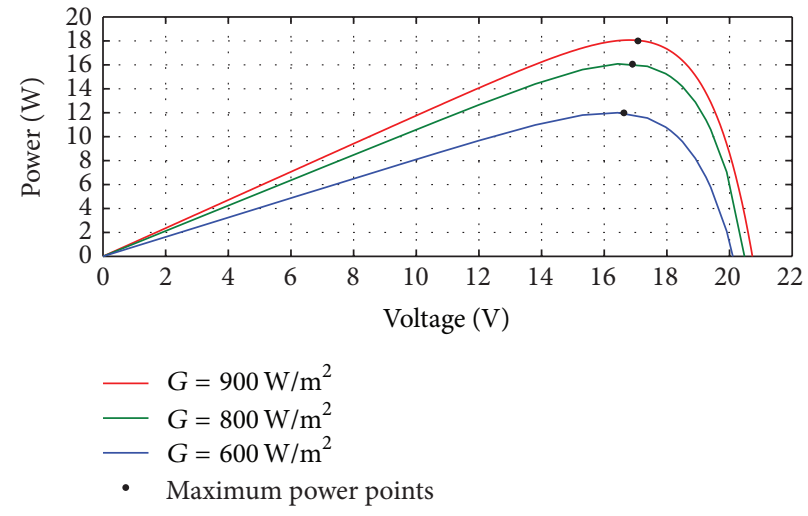

(b)

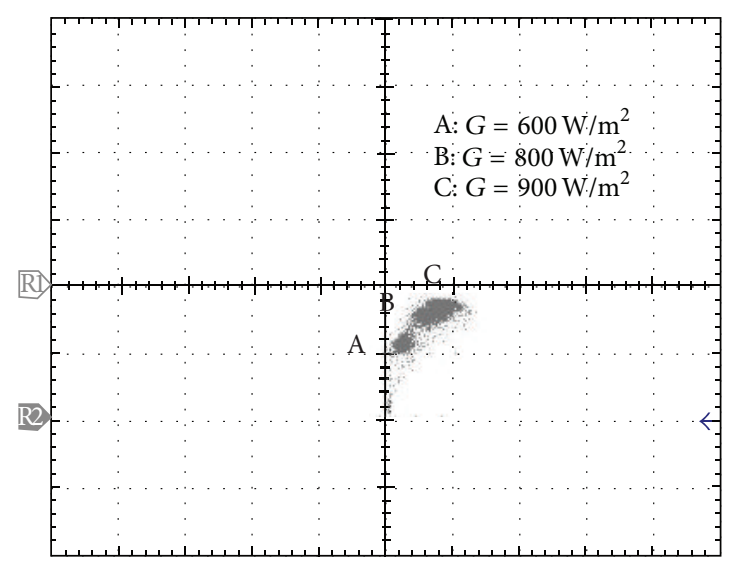

(c)

FIGURE 8: (a) Experimental waveforms of power, voltage, and current for TBP-1237 module (Y-Axis-V/div:10 V/div;A/div:1 A/div;W/div:10 W/ div), (b) simulation PV waveforms, and (c) experimental power points from MPP tracking using P and O method.

from the actual curve between the MPP and the open circuit point. Extracting the advantage of the above two models, an improved simplified single diode model has been proposed here, where the result closely matches not only at the three points: the open circuit point, the MPP, and the short circuit point, but also throughout the curve.

4.2. Analysis on Basis of Simulation Time. The simulation time taken by the models has been shown in Table 4. More simulation time indicates a higher complexity of the model. As expected, the single diode model takes the highest time on account of the computational complexity offered by it. It is then followed by the proposed model and the lowest time is taken by the improved ideal single diode model. However, the time difference offered by the latter ones is very less and hence no significant time is elapsed during simulation.

4.3. Analysis of Basis of Fill Factor. Another factor which determines the quality of a model is the fill factor. This factor gives the ratio of the power produced at the maximum power point to the maximum theoretical power that should be possible to extract from the module. For good cells, the value should generally be more than 0.7 . It is known that the higher the fill factor, the better the model. Table 4 shows the fill factors for various models. Much difference is not observed between the fill factors. However, it is seen that the proposed model gives the highest fill factor. It is followed by the single diode model and the least fill factor is seen in the improved ideal single diode model. It is due to its deviation in the curve from the actual experimental data. Although the curve at the MPP deviates towards the lower side in case of single diode model, it shows a higher fill factor on account of its greater deviation towards the higher side at the other regions.

4.4. Analysis on Basis of Maximum Efficiency. The subsequent parameter used for the analysis is the maximum efficiency which is the ratio between the maximum power and the incident power. At an irradiation of $1000 \mathrm{~W} / \mathrm{m}^{2}$ and a temperature of $35^{\circ} \mathrm{C}$, the maximum efficiency offered by the models in $\%$ is illustrated in Table 4 .

4.5. Analysis on Basis of RMSD. The accuracy of a particular model can be known from the RMSD it shows. Higher the RMSD value, less accurate the model. Table 4 shows the RMSD comparison between the various PV models. The least RMSD is shown by the proposed model, since it takes 


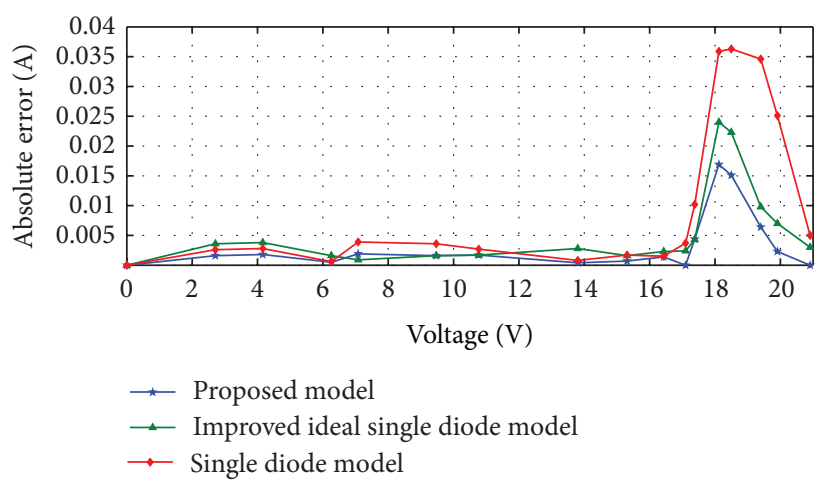

(a)

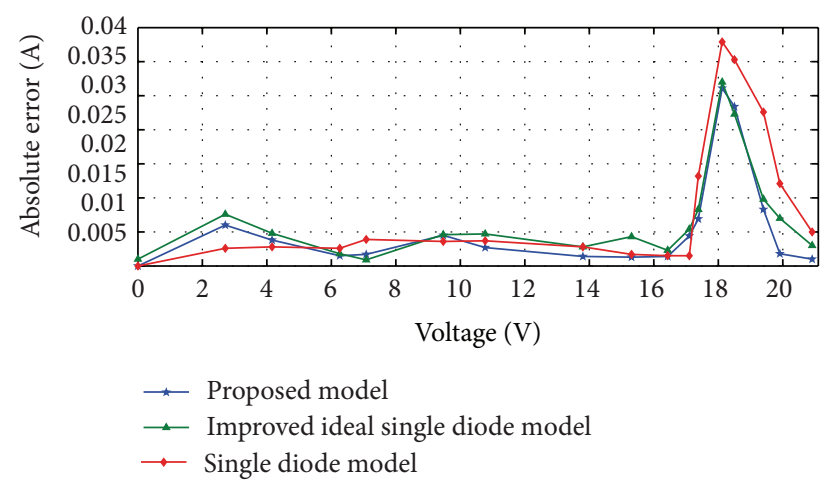

(b)

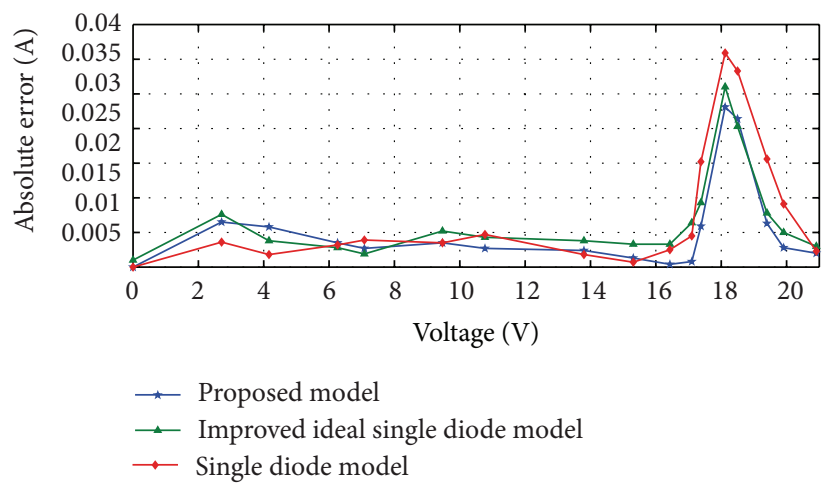

(c)

Figure 9: Comparison of absolute errors among three different approaches for TBP-1237 module at (a) STC (Temp: 25 C; A.M.: 1.5; $G=$ $1000 \mathrm{~W} / \mathrm{m}^{2}$ ), (b) Temp: $35^{\circ}$ C; A.M.: $1.5 ; G=\mathrm{W} / \mathrm{m}^{2}$, and (c) Temp: $35^{\circ}$ C; A.M.: $1.5 ; G=1000 \mathrm{~W} / \mathrm{m}^{2}$.

into account all the parameters. The improved ideal single diode model also shows a low RMSD value even if some of the parameters are ignored since it is not based on any assumption in calculation. The single diode model shows a better RMSD than the improved ideal single diode model. However, it is not true in all cases. In some cases of varying temperature and irradiation, the improved ideal single diode model proves to be a better model than the single diode model. The absolute errors for these models are compared in Figure 9(a) for STC. It is seen that the proposed model provides better results when compared with the results found by different approaches for the same module.

In order to verify the notion that the proposed model gives better accuracy, even far from STC, comparisons have been made based on the absolute values of error as shown in Figures 9(b) and 9(c).

4.6. Experimental Validation for MPP Tracking. The proposed method being an improvement over the conventional method and exhibiting closeness to the real cell at MPP is validated by comparing with the experimental result. Figure 7 depicts the prototype used with PV panel as the input to the converter and battery of $45 \mathrm{~W}$ as its output. The current and voltage sensed by the sensors are used to generate duty ratio in the FPGA environment. With battery as the output of the converter, the input is regulated by the $\mathrm{P} \& \mathrm{O}$ control algorithm where irrespective of the alteration in irradiation MPP point for each $P-V$ curve is tracked efficiently. Figure 8 shows the experimental waveform of the MPP points for three different irradiation levels which perfectly matches with the one found from the modeling.

\section{Conclusions}

This paper analyses the development of an effective approach to modeling of PV modules used for simulation purposes. The proposed modeling fits the mathematical characteristic model equations to the experimental curve. In addition to it, it avoids complexities and achieves better accuracy by the inclusion of the series resistance. The dependency of diode saturation current on the temperature as well as on the MPP helps in making the equation effective and allows the user to adjust the output curve at the open-circuit voltage, short circuit current point, and the MPP at different temperatures and irradiations. Also, the inclusion of the calculation of the ideality factor from the information available in the datasheet improves the accuracy of the curve and fits the curve with the experimental one. The results are experimentally validated to prove the effectiveness of the proposed method. The Perturb and Observe MPPT algorithm implemented along with the boost converter at different irradiation substantiate the closeness of the MPP points of the proposed model to 
TABLE 4: Parameters used for comparative analysis.

\begin{tabular}{lccccc}
\hline Model & MPP points & RMSD & FF & Max. eff. (in \%) & Simulation time \\
\hline Single diode Model & $\begin{array}{c}17.092 \mathrm{~V} \\
1.172 \mathrm{~A}\end{array}$ & 0.0561 & 0.7256 & 15.7366 & $2.14 \mathrm{~s}$ \\
\hline Improved ideal single diode model & $\begin{array}{c}17.087 \mathrm{~V} \\
1.169 \mathrm{~A}\end{array}$ & 0.0587 & 0.7244 & 15.6881 & $0.86 \mathrm{~s}$ \\
\hline Proposed model & $\begin{array}{l}17.1 \mathrm{~V} \\
1.17 \mathrm{~A}\end{array}$ & 0.0531 & 0.7263 & 15.7862 & $0.89 \mathrm{~s}$ \\
\hline
\end{tabular}

the real time observed points. Along with it, the comparison of the proposed model with the conventional along with the improved single diode model in terms of the fundamental quantities such as fill factor, RMSD, and maximum efficiency gives a better insight into the advantage of the proposed model to the previous ones.

The proposed mathematical model is easy to implement in various simulation platforms for PV power system studies and it avoids the underlying complexities involved in PV parameter identification. This paper is intended to be a useful tool, serving the cause of both beginners as well as seasoned users.

\section{Conflict of Interests}

The authors declare that there is no conflict of interests regarding the publication of this paper.

\section{References}

[1] M. A. G. de Brito, L. P. Sampaio, L. G. Junior, and C. A. Canesin, "Evaluation of MPPT techniques for photovoltaic applications," in Proceedings of the IEEE International Symposium on Industrial Electronics (ISIE '11), pp. 1039-1044, Gdansk, Poland, June 2011.

[2] M. G. Villalva, J. R. Gazoli, and E. R. Filho, "Comprehensive approach to modeling and simulation of photovoltaic arrays," IEEE Transactions on Power Electronics, vol. 24, no. 5, pp. 11981208, 2009.

[3] M. Sokolov, T. C. Green, P. D. Mitcheson, and D. Shmilovitz, "Dynamic analysis of photovoltaic system with MPP locus emulation," in Proceedings of the IEEE 26th Convention of Electrical and Electronics Engineers in Israel (IEEEI '10), pp. 212215, Eliat, Israel, November 2010.

[4] G. Walker, "Evaluating MPPT converter topologies using a matlab PV model," Journal of Electrical \& Electronics Engineering, Australia, vol. 21, no. 1, pp. 49-55, 2001.

[5] D. S. H. Chan and J. C. H. Phang, "Analytical methods for the extraction of solar-cell single- and double-diode model parameters from I-V characteristics," IEEE Transactions on Electron Devices, vol. 34, no. 2, pp. 286-293, 1987.

[6] J. A. Gow and C. D. Manning, "Development of a photovoltaic array model for use in power-electronics simulation studies," IEE Proceedings: Electric Power Applications, vol. 146, no. 2, pp. 193-200, 1999.

[7] G. E. Ahmad, H. M. S. Hussein, and H. H. El-Ghetany, "Theoretical analysis and experimental verification of PV modules," Renewable Energy, vol. 28, no. 8, pp. 1159-1168, 2003.
[8] M. Veerachary, "PSIM circuit-oriented simulator model for the nonlinear photovoltaic sources," IEEE Transactions on Aerospace and Electronic Systems, vol. 42, no. 2, pp. 735-740, 2006.

[9] A. N. Celik and N. Acikgoz, "Modelling and experimental verification of the operating current of mono-crystalline photovoltaic modules using four- and five-parameter models," Applied Energy, vol. 84, no. 1, pp. 1-15, 2007.

[10] Y.-C. Kuo, T.-J. Liang, and J.-F. Chen, "Novel maximum-powerpoint-tracking controller for photovoltaic energy conversion system," IEEE Transactions on Industrial Electronics, vol. 48, no. 3, pp. 594-601, 2001.

[11] M. T. Elhagry, A. A. T. Elkousy, M. Saleh, T. Elshatter, and E. M. Abou-Elzahab, "Fuzzy modeling of photovoltaic panel equivalent circuit," in Proceedings of the 40th Midwest Symposium on Circuits and Systems, vol. 1, pp. 60-63, August 1997.

[12] S. Liu and R. A. Dougal, "Dynamic multiphysics model for solar array," IEEE Transactions on Energy Conversion, vol. 17, no. 2, pp. 285-294, 2002.

[13] D. Sera, R. Teodorescu, and P. Rodriguez, "PV panel model based on datasheet values," in Proceedings of the IEEE International Symposium on Industrial Electronics (ISIE '07), pp. 23922396, Vigo, Spain, June 2007.

[14] M. A. Vitorino, L. V. Hartmann, A. M. N. Lima, and M. B. R. Corrêa, "Using the model of the solar cell for determining the maximum power point of photovoltaic systems," in Proceedings of the European Conference on Power Electronics and Applications (EPE '07), pp. 1-10, Aalborg, Denmark, September 2007.

[15] H. Patel and V. Agarwal, "MATLAB-based modeling to study the effects of partial shading on PV array characteristics," IEEE Transactions on Energy Conversion, vol. 23, no. 1, pp. 302-310, 2008.

[16] R. Noroozian, M. Abedi, G. Gharehpetian, and S. Hosseini, Modelling and simulation of microturbine generation system for on-grid and offgrid operation modes.

[17] S. Bal, A. Anurag, and B. C. Babu, "Comparative analysis of mathematical modeling of Photo-Voltaic (PV) array," in Proceedings of the Annual IEEE India Conference (INDICON '12), pp. 269-274, Kochi, India, December 2012.

[18] Y. T. Tan, D. S. Kirschen, and N. Jenkins, "A model of PV generation suitable for stability analysis," IEEE Transactions on Energy Conversion, vol. 19, no. 4, pp. 748-755, 2004.

[19] A. Kajihara and T. Harakawa, "Model of photovoltaic cell circuits under partial shading," in Proceedings of the IEEE International Conference on Industrial Technology (ICIT'05), pp. 866-870, December 2005.

[20] M. Glass, "Improved solar array power point model with spice realization," in Proceedings of the 31st Intersociety Energy Conversion Engineering Conference (IECEC '96), vol. 1, pp. 286291, August 1996. 
[21] I. H. Altas and A. M. Sharaf, "A photovoltaic array simulation model for matlab-simulink GUI environment," in Proceedings of the International Conference on Clean Electrical Power (ICCEP '07), pp. 341-345, Capri, Italy, May 2007.

[22] Z. Salam, K. Ishaque, and H. Taheri, "An improved two-diode photovoltaic (PV) model for PV system," in Proceedings of the Joint International Conference on Power Electronics, Drives and Energy Systems \& Power India (PEDES '10), pp. 1-5, IEEE, New Delhi, India, December 2010.

[23] Y. Mahmoud, W. Xiao, and H. H. Zeineldin, "A simple approach to modeling and simulation of photovoltaic modules," IEEE Transactions on Sustainable Energy, vol. 3, no. 1, pp. 185-186, 2012.

[24] D. Bonkoungou, Z. Koalaga, and D. Njomo, "Modelling and simulation of photovoltaic module considering single-diode equivalent circuit model in MATLAB," International Journal of Emerging Technology and Advanced Engineering, vol. 3, no. 3, pp. 493-502, 2013.

[25] M. Hatti, A. Meharrar, and M. Tioursi, "Novel approach of maximum power point tracking for photovoltaic module neural network based," in Proceedings of the International Symposium on Environment Friendly Energies in Electrical Applications, pp. 1-6, Ghardaïa, Algeria, November 2010.

[26] K. Nishioka, N. Sakitani, Y. Uraoka, and T. Fuyuki, "Analysis of multicrystalline silicon solar cells by modified 3-diode equivalent circuit model taking leakage current through periphery into consideration," Solar Energy Materials and Solar Cells, vol. 91, no. 13, pp. 1222-1227, 2007.

[27] S. Bal, A. Anurag, and B. C. Babu, "Comparative analysis of mathematical modeling of Photo-Voltaic (PV) array," in Proceedings of the Annual IEEE India Conference (INDICON '12), pp. 269-274, Kochi, India, December 2012.

[28] S. Chowdhury, S. P. Chowdhury, G. Taylor, and Y. H. Song, "Mathematical modelling and performance evaluation of a stand-alone polycrystalline pv plant with mppt facility," in Proceedings of the 2008 IEEE Power and Energy Society General Meeting-Conversion and Delivery of Electrical Energy in the 21st Century, pp. 1-7, July 2008.

[29] W. de Soto, S. A. Klein, and W. A. Beckman, "Improvement and validation of a model for photovoltaic array performance," Solar Energy, vol. 80, no. 1, pp. 78-88, 2006.

[30] M. Sabry and A. E. Ghitas, "Influence of temperature on methods for determining silicon solar cell series resistance," Journal of Solar Energy Engineering, vol. 129, no. 3, pp. 331-335, 2007.

[31] G. H. Yordanov, O.-M. Midtgård, and T. O. Saetre, "Equivalent cell temperature calculation for PV modules with variable ideality factors," in Proceedings of the 38th IEEE Photovoltaic Specialists Conference (PVSC '12), pp. 505-508, Austin, Tex, USA, June 2012. 

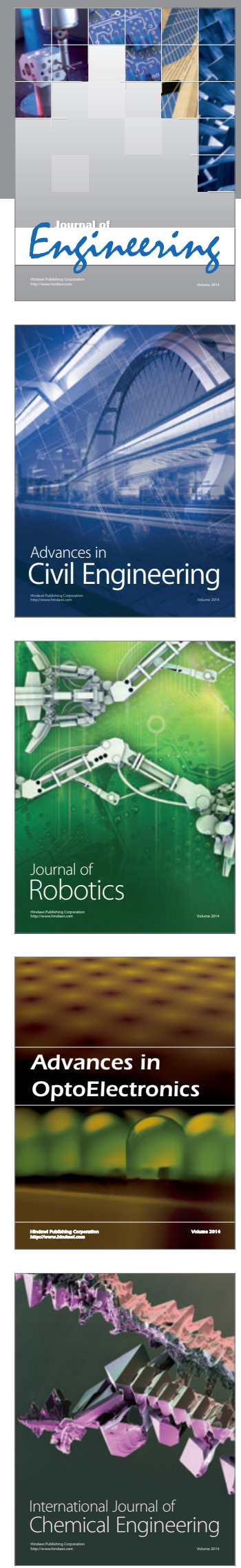

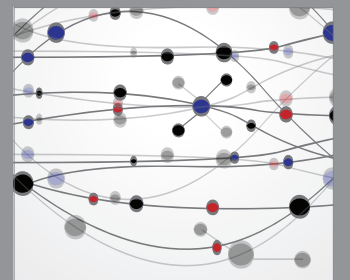

The Scientific World Journal
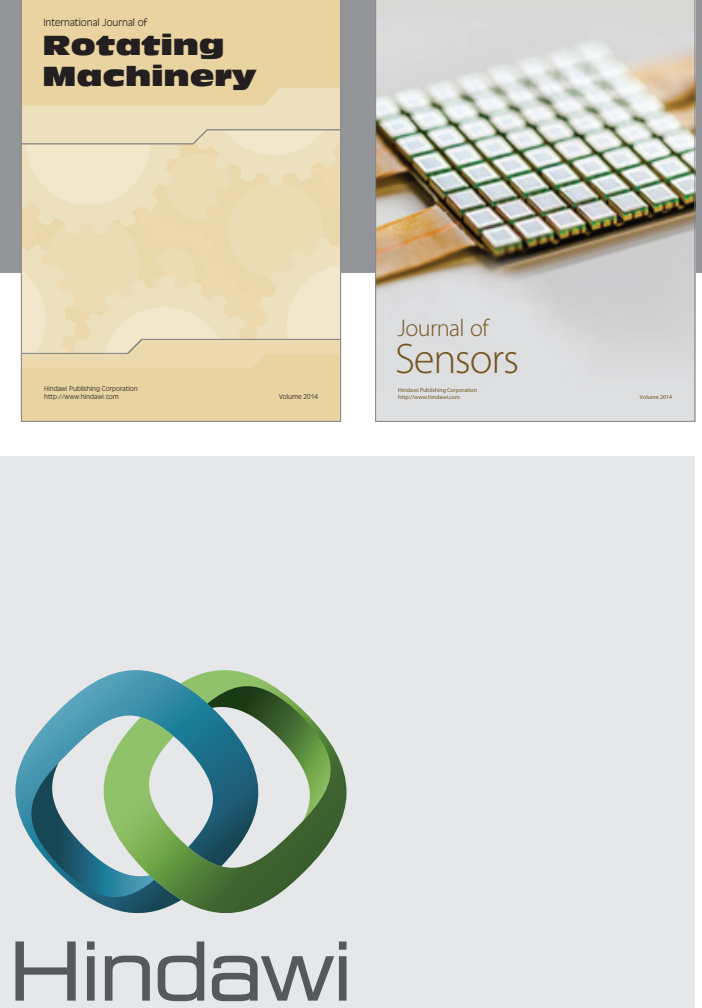

Submit your manuscripts at http://www.hindawi.com
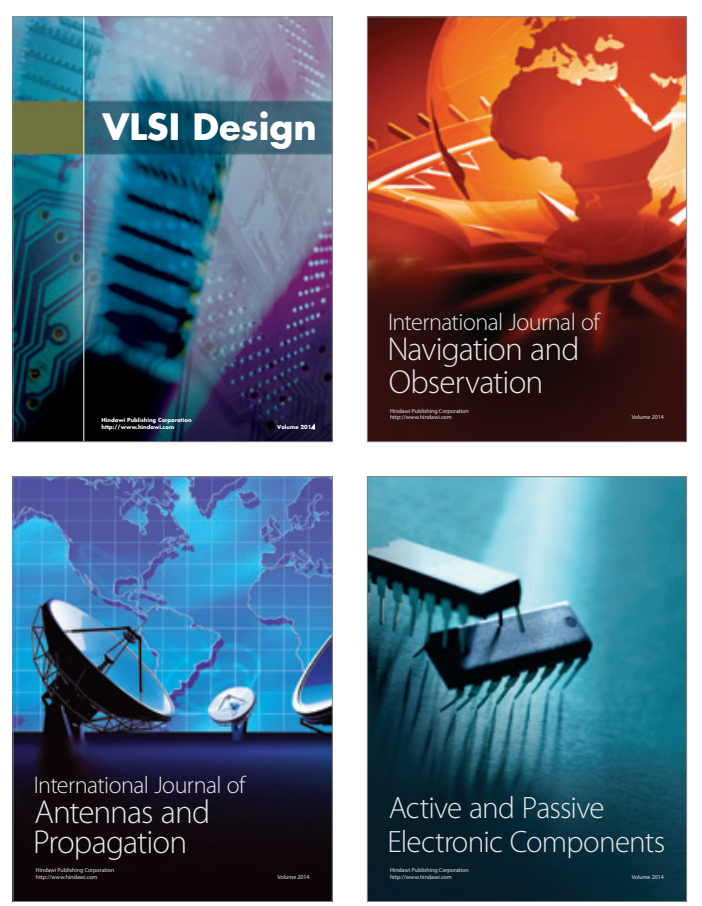
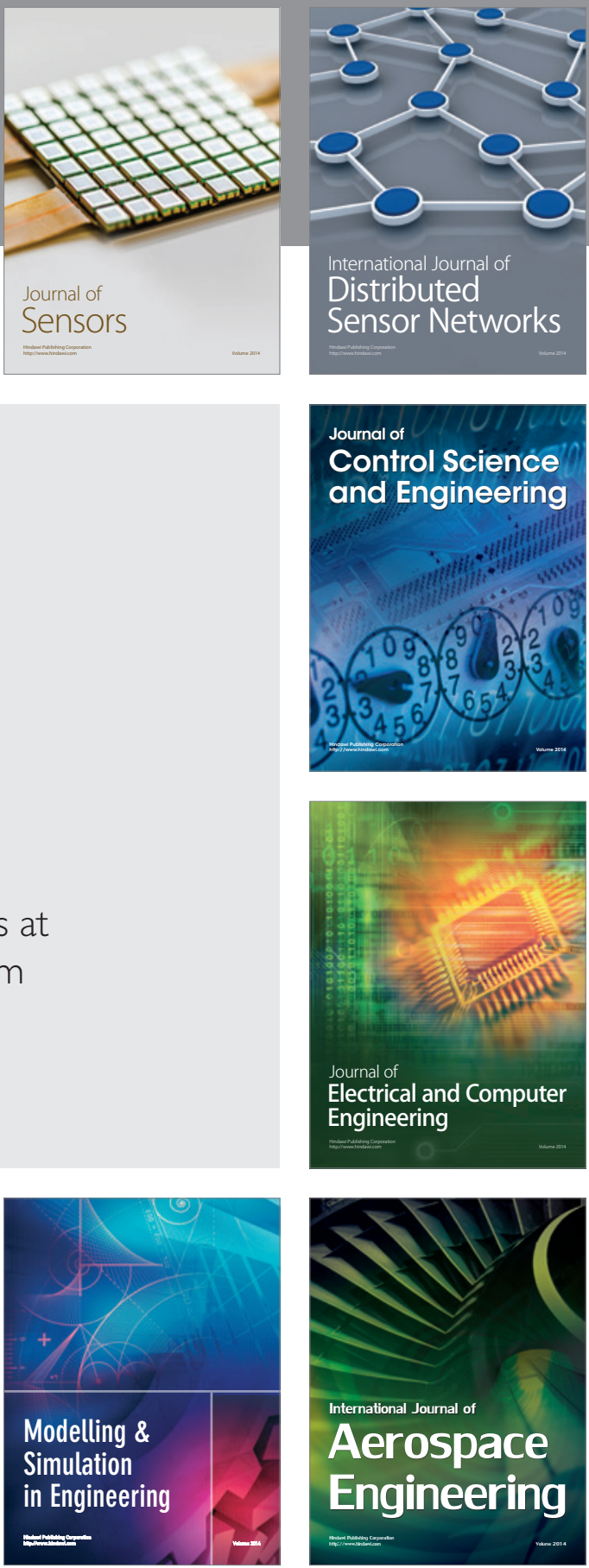

Journal of

Control Science

and Engineering
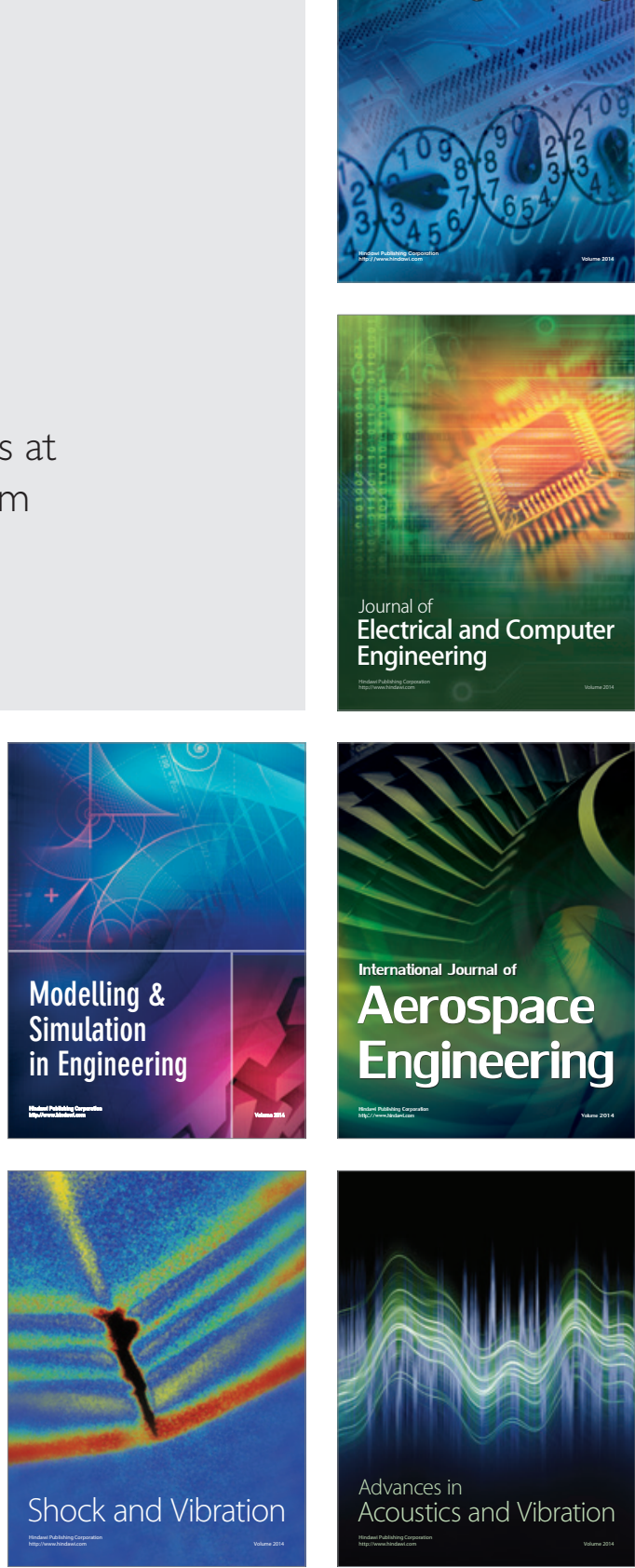